\title{
Design and synthesis of tetrahydrophthalimide derivatives as inhibitors of HIV-1 reverse transcriptase
}

\author{
Ashok Penta', Swastika Ganguly ${ }^{2}$ and Sankaran Murugesan ${ }^{1 *}$
}

\begin{abstract}
Background: Non-nucleoside reverse transcriptase inhibitors (NNRTIs) are one of the key components in highly active anti-retroviral therapy because of their high specificity and less toxicity. NNRTIs inhibit reverse transcriptase enzyme by binding to the allosteric site, which is $10 \AA$ away from the active site. Rapid emergence of resistance is the major problem with all anti-HIV agents. Hence, there is continuous need to develop novel anti-HIV agents active against both drug sensitive and resistance strains.

Results: All the 16 synthesized 2-(1,3-dioxo-3a,4-dihydro-1H-isoindol-2(3H,7H,7aH)-yl)-N-(substitutedphenyl) acetamide 4(a-p) analogs were characterized by Fourier transform infrared spectroscopy, proton nuclear magnetic resonance spectroscopy, mass spectroscopy, and elemental analysis. Lipinski rule of five parameters and molecular parameters like solubility, drug likeness, and drug score were derived for designed analogs using online servers like Molinspiration and Osiris property explorer. Synthesized compounds were evaluated for their HIV-1 reverse transcriptase inhibitor activity by HIV-1 RNA-dependent DNA polymerase activity assay at 2 and $20 \mu \mathrm{M}$ concentrations.
\end{abstract}

Conclusions: Among the 16 synthesized compounds, 4a, 4b, 4f, 4g, 4k, and $4 \mathrm{l}$ showed weak reverse transcriptase inhibitor activity at $20 \mu \mathrm{M}$ concentration. For the designed compounds, there was no correlation observed between molecular modeling and in vitro studies.

Keywords: NNRTIs; HAART; HIV-1 reverse transcriptase; Docking; Molecular properties; Autodock; Tetrahydrophthalimide

\section{Background}

Acquired immune deficiency syndrome (AIDS) is the advance stage of infection caused by the human immunodeficiency virus (HIV-1). AIDS and AIDS-ailed infections are major leading causes of death. According to UNAIDS-2012 report, 33 million people are living with AIDS and 1.7 million people died in the year 2011 [1]. Highly active antiretroviral therapy (HAART), a combination of two nucleotide or nucleoside reverse transcriptase inhibitor (NRTIs) and one protease inhibitor (PI), is generally used for AIDS. Alternative combinations like two NRTIs and one nonnucleoside reverse transcriptase inhibitor (NNRTI) or two NRTIs and one integrase inhibitor are used. NNRTIs are

\footnotetext{
* Correspondence: murugesaa789@gmail.com

${ }^{1}$ Department of Pharmacy, Birla Institute of Technology \& Science, Pilani Rajasthan 333031, India

Full list of author information is available at the end of the article
}

the key components in HAART because of their high potency, selectivity, and less toxicity when compared to NRTIs and PIs [2,3]. Currently five NNRTIs are approved by United States Food and Drug Administration. Among them, nevirapine, delavirdine, and efavirenz are first generation, which already got resistance. Etravirine and rilpivirine are potent and currently using second generation NNRTIs. However, the occurrence of the high mutation rate of the virus and the resulting emergence of resistance make the researchers run a never-ending marathon to keep developing new drugs active against both drug-sensitive and drug-resistance strain with better therapeutic profile $[4,5]$.

Many NNRTIs, including tetrahydroimidazo [4,5,1-jkj] $[1,4]$ benzodiazepin-2(1H)-one and $\alpha$-anilinophenyl acetamide derivatives, adapt typical butterfly-like conformations in non-nucleoside inhibitory binding pocket (NNIBP), with 
one hydrophilic body and two hydrophobic wings (wing-1 and wing-2). Hydrophilic body contains mainly functional groups like $-\mathrm{NH},-\mathrm{C}=\mathrm{O}$, and $-\mathrm{OH}$ which are able to form hydrogen bonding interactions with active site amino acids like K101, K103, and P236. Hydrophobic wings are $\pi$-electron containing aromatic ring system, which can form hydrophobic interactions and pi-cationic interactions with amino acids Y181, Y188, W229, F227, V106, P236, L100, L234, and Y318 [6,7].

Compounds having phthalimide scaffold exhibit antiinflammatory [8], anticancer [9], antibacterial [10], HIV-1 RT [11,12], and HIV-1 integrase inhibitory [13] activities. An extensive perusal of literature revealed that little work has been done on phthalimides and tetrahydrophthalimide as NNRTIs. In view of these facts and our interest on the development of novel NNRTIs, we have chosen tetrahydrophthalimide scaffold as one of the hydrophobic wings in butterfly-shape pharmacophore. All the newly synthesized compounds were designed based on the derived pharmacophoric model with acetamide moiety as hydrophilic body, and tetrahydrophthalimide and substituted aromatic amines as hydrophobic wings (general structure shown in Figure 1).

\section{Methods}

\section{Molecular docking study}

The docking studies of all the derivatives $4(\mathbf{a}-\mathbf{p})$ were performed using molecular modeling software Autodock 4.2 (The Scripps Research Institute, CA, USA) [14] installed on a single machine running on a 3.4-GHz Pentium processor with Windows XP SP2 as the operating system. HIV-1 RT enzyme (pdb code 1rt2 (shown in Figure 2)) was taken from the RCSB, used as target protein [7]. Target protein $\mathrm{pdb}$ was further refined by removal of water molecules and by adding polar hydrogens and Kollmancharges. For the docking, a grid spacing of $0.375 \AA$ and $63 \times 63 \times$ 63 number of points were used. The grid was centered on the active site. The auto grid program generated separate

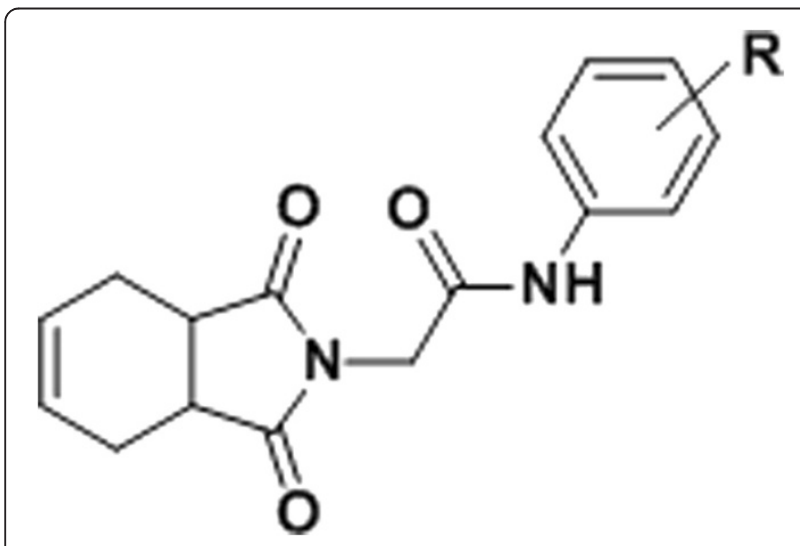

Figure 1 General structure of synthesized compounds.

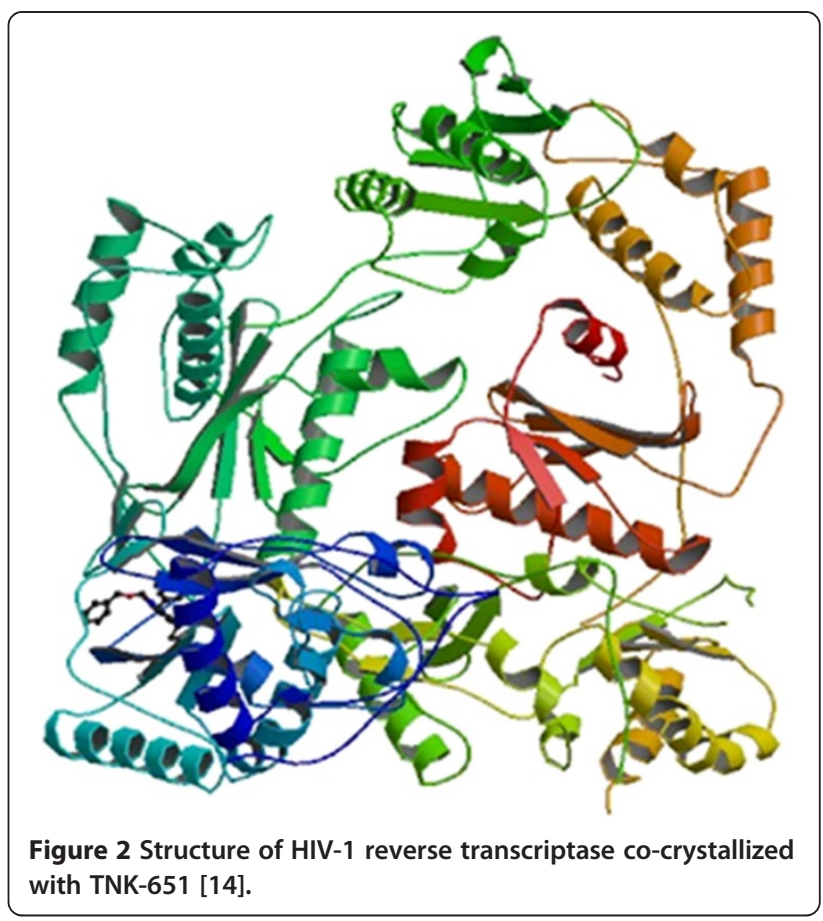

grid maps for all atom types of the ligand structures and one for electrostatic interactions. PRODRG online server was used to generate the energy minimized conformations of the ligands in pdb format [15]. Energy minimized conformation of ligands was subjected to calculation of Gasteiger-Huckel charges and saved in default format of Autodock. Autodock generated 50 possible binding conformations, i.e., 50 runs for each docking by using LGA search. Default protocol was applied, with initial population of 150 randomly placed individuals, a maximum number of $2.5 \times 10^{5}$ energy evaluations and $2.7 \times 10^{4}$ generations. A mutation rate of 0.02 and a crossover rate of 0.8 were used.

\section{Validation of docking}

Initially, the receptor was docked with extracted ligand TNK 651 in order to validate the docking calculations, reliability, and reproducibility of the docking parameters for the study. It was evident that the docked pose of the re-docked ligand was almost superimposed with that of the co-crystallized ligand (Figure 3 ) with RMSD value of 0.5. Then, docking was performed with the standard drug efavirenz with 1 tr 2 for validation, and the mode of interaction was shown in Figure 4.

The binding free energies (docking score) and predicted inhibitory constant (Ki) values of the designed analogs were compared with binding free energies and inhibitory constants of the co-crystallized ligand TNK-651 and standard drug efavirenz. Binding free energies and predicted inhibitory constant values of TNK-651, efavirenz, and designed analogs were given in Table 1. Docking studies of 


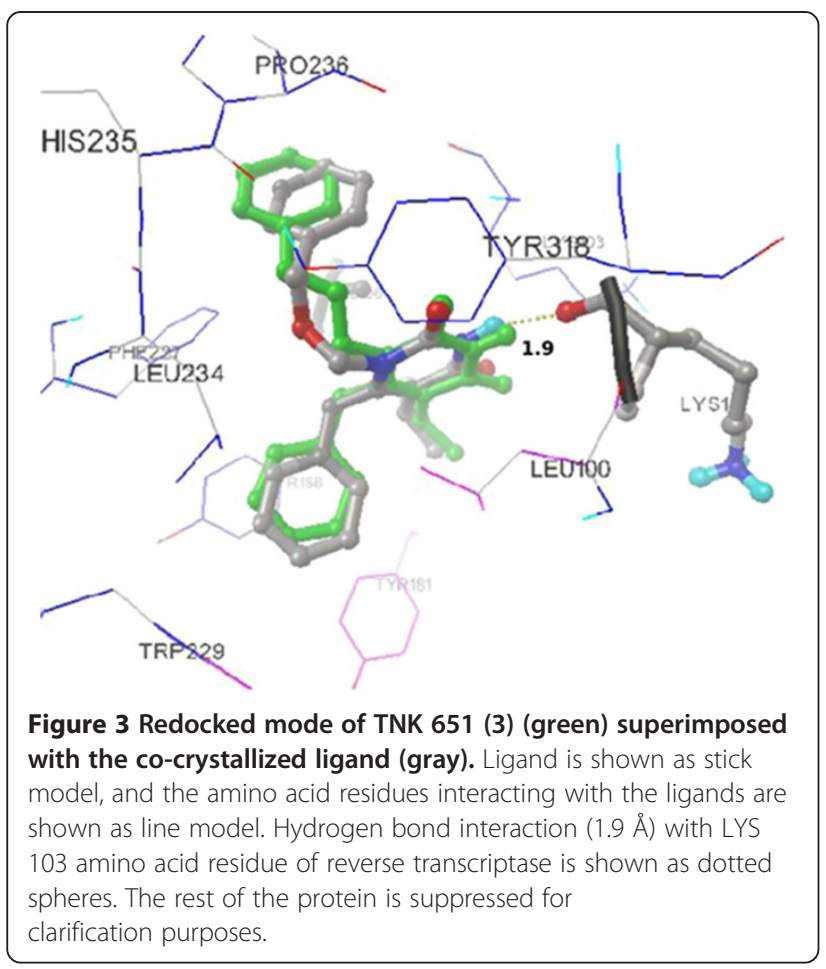

designed compounds have shown satisfactory results. Hydrophilic body of designed analogs showed hydrogen bonding interactions with amino acids of receptor protein 1rt2. Hydrogen bonding interactions of compound 41 with LYS 101 and LYS 103 are shown in Figure 5. All the

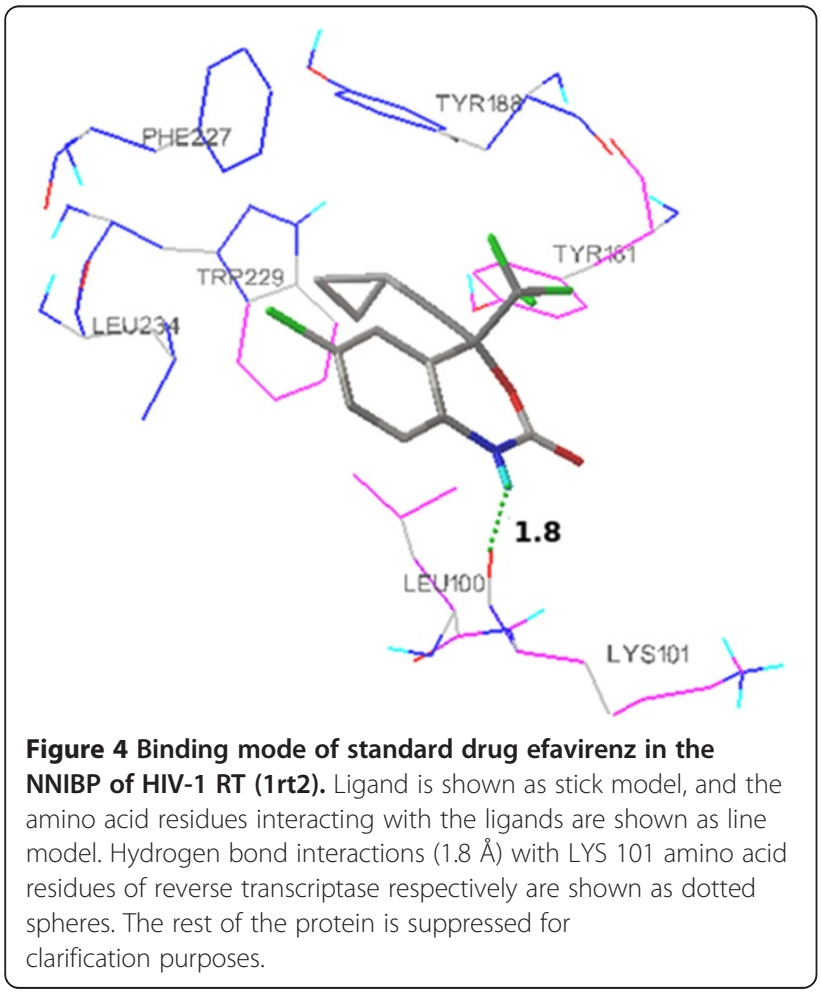

Table 1 Binding free energy and predicted inhibitory constant values of the synthesized compounds

\begin{tabular}{|c|c|c|c|c|}
\hline $\begin{array}{l}\text { Serial } \\
\text { number }\end{array}$ & $\begin{array}{l}\text { Compound } \\
\text { code }\end{array}$ & $R$ & $\begin{array}{l}\text { Binding } \\
\text { free energy } \\
\text { (Kcal/mole) }\end{array}$ & $\begin{array}{l}\text { Inhibitory } \\
\text { constant (nM) }\end{array}$ \\
\hline 1 & $\begin{array}{l}\text { Efavirenz } \\
\text { (standard) }\end{array}$ & - & -12.02 & 1.56 \\
\hline 2 & TNK-651 & - & -11.88 & 1.95 \\
\hline 3 & $4 a$ & $\mathrm{H}$ & -8.13 & $1,100.0$ \\
\hline 4 & $4 b$ & $4-\mathrm{OCH}_{3}$ & -7.65 & $2,460.0$ \\
\hline 5 & $4 c$ & $4-\mathrm{CH}_{3}$ & -8.5 & 588.3 \\
\hline 6 & $4 d$ & $4-\mathrm{Cl}$ & -7.79 & $1,930.0$ \\
\hline 7 & $4 e$ & $3-\mathrm{OCH}_{3}$ & -8.62 & 479.1 \\
\hline 8 & $4 f$ & $3-\mathrm{CH}_{3}$ & -8.61 & 484.7 \\
\hline 9 & $4 g$ & $3-\mathrm{Cl}$ & -8.87 & 315.7 \\
\hline 10 & $4 \mathrm{~h}$ & $2-\mathrm{OCH}_{3}$ & -7.94 & $1,510.0$ \\
\hline 11 & $4 i$ & $2-\mathrm{CH}_{3}$ & -8.2 & 975.83 \\
\hline 12 & $4 j$ & $2-\mathrm{Cl}$ & -8.2 & 975.83 \\
\hline 13 & $4 k$ & $4-\mathrm{NO}_{2}$ & -10.53 & 19.11 \\
\hline 14 & 41 & $3-\mathrm{NO}_{2}$ & -10.89 & 10.38 \\
\hline 15 & $4 m$ & $2-\mathrm{NO}_{2}$ & 10.46 & 22.86 \\
\hline 16 & $4 n$ & $2,4-\mathrm{diCH}_{3}$ & -8.87 & 313.63 \\
\hline 17 & 40 & $3,4-\mathrm{diCH}_{3}$ & 10.49 & 20.37 \\
\hline 18 & $4 p$ & $2-\mathrm{Cl}, 3-\mathrm{CH}_{3}$ & -10.71 & 14.2 \\
\hline
\end{tabular}

$4 k, 4 l, 4 m, 40$, and $4 p$ showed satisfactory and comparable docking results as that of standard drug efavirenz and TNK-651 (the same thing has been discussed under the subsection "In vitro HIV-1 RT inhibitory activity" of the "Results and discussion" section).

designed analogs showed similar orientation in NNIBP of receptor protein. The orientation of some designed compounds (having low binding free energy) in NNIBP of receptor is shown in Figure 6.

\section{Molecular parameters}

Lipinski rule of five parameters like ClogP, molecular weight, number of hydrogen bond acceptors (HBA), number of hydrogen bond donors (HBD), solubility, drug likeness, and drug score were derived through online servers Molinspiration (Molinspiration Cheminformatics, Nova Ulica, Slovak Republic) and OSIRIS (Organic Chemistry, Switzerland) property calculator [16,17]. All the calculated values were given in Table 2.

\section{Results and discussion \\ Chemistry}

Designed analogs were synthesized using a synthetic protocol shown in Scheme 1. In the first step, 2-chloro- $N$ (substituted phenyl) acetamide (2a-p) analogs were synthe sized by treating substituted anilines with 2-chloroacetyl chloride in dichloromethane and triethylamine as base. 2chloro- $N$-(substituted phenyl) acetamide (2a-p) intermediates were then treated with tetrahydrophthalimide (3) 


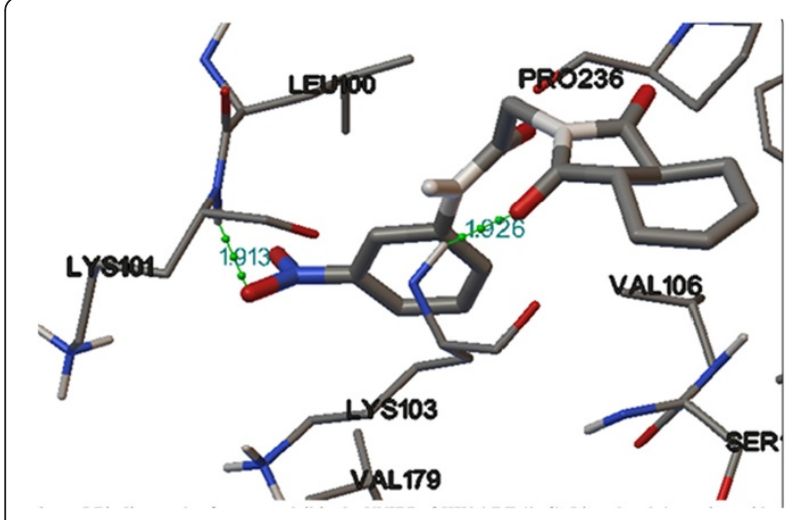

Figure 5 Binding mode of compound $4 \mathrm{l}$ in the NNIBP of HIV-1 RT (1rt2). Ligand and the amino acid residues interacting with the ligands are shown as ball-and-sticks model. Hydrogen bond interactions (1.913 $\AA$ ) with LYS 101 and (1.926 $\AA$ ) with LYS 103 amino acid residues of reverse transcriptase are shown as dotted spheres. The rest of the protein is suppressed for clarification purposes.

in presence of base potassium carbonate and acetonitrile as solvent to yield titled compounds as final products $\mathbf{4}(\mathbf{a}-\mathbf{p})[18,19]$.

Synthesized compounds were isolated as pure and characterized by IR, ${ }^{1} \mathrm{H}$ NMR, mass, and elemental analysis data. In general, the IR spectra of the synthesized compounds showed $\mathrm{N}-\mathrm{H}$ stretching at around 3,408 to $3,259 \mathrm{~cm}^{-1}, \mathrm{C}=\mathrm{O}$ (amide) absorption band at around 1,703 to $1,682 \mathrm{~cm}^{-1}, \mathrm{C}=\mathrm{O}$ (phthalimide) absorption band at around 1,786 to 1,768 and 1,712 to $1,702 \mathrm{~cm}^{-1}$, C-O-C absorption (methoxy) band at around 1,249 to $1,234 \mathrm{~cm}^{-1}$, and $\mathrm{C}-\mathrm{Cl}$ absorption band at around 697 to $678 \mathrm{~cm}^{-1}$. The ${ }^{1} \mathrm{H}$ NMR spectrum of the product $4 \mathrm{c}$ (see 'Experimental' section) showed two characteristic

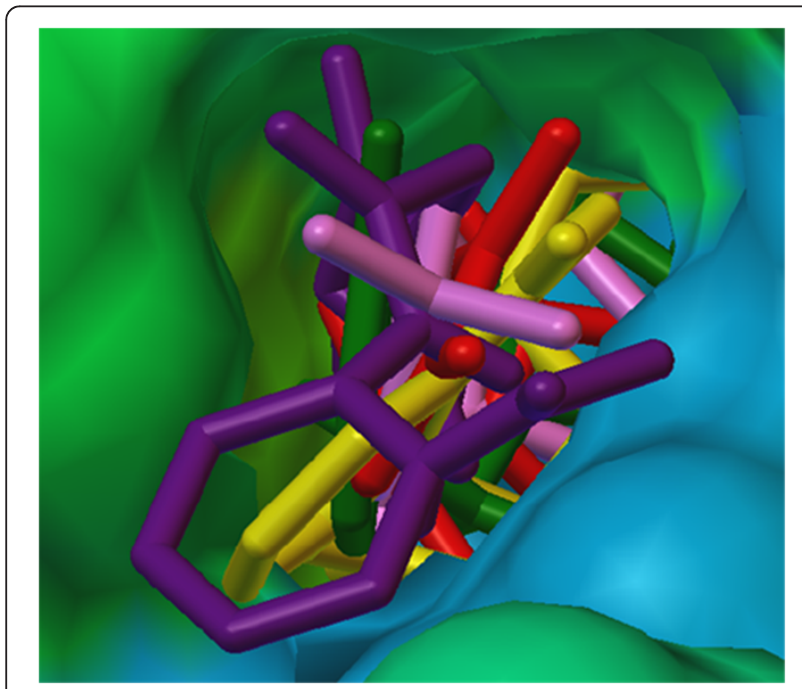

Figure 6 Overlay stereoview. 4k (pink), 4l (yellow), 4m (violet), 40 (red), and $4 p$ (green)in the NNIBP of HIV-1 RT. singlets at $\delta 4.27$ and $\delta 2.30$ because of $\mathrm{COCH}_{2}-\mathrm{N}$ and $\mathrm{CH}_{3}$, respectively. One broad singlet at $\delta 7.36$ indicates the presence of $\mathrm{NH}$, two doublets at $\delta 7.32$, and $\delta 7.10$ confirms the presence of para-substituted benzene ring. Besides these, the aliphatic region also showed the characteristic multiplet peaks due to $\mathrm{CH}=\mathrm{CH}, \mathrm{CH}$ $\mathrm{CH}$, and $=\mathrm{CH}-\mathrm{CH}_{2}$ at $\delta 5.96$ to $5.97, \delta 3.21$ to $3.23, \delta$ 2.63 to 2.69 , and $\delta 2.26$ to 2.28 , respectively. Mass spectral analysis of the compounds4a and $4 \mathbf{c}$ showing the molecular ion peak at 285.6 and $299.6(M+1)$, respectively, confirms the molecular weight of the desired compounds.

\section{In vitro HIV-1 RT inhibitory activity}

All the synthesized compounds $\mathbf{4}(\mathbf{a}-\mathbf{p})$ were evaluated for HIV-1 RT inhibitory activity at concentrations 2 and $20 \mu \mathrm{M}$ by using HIV-1 RT RNA-dependent DNA polymerase activity assay [20]. HIV-1 RT inhibitory activity results are shown in Table 3. Rilpivirine was used as standard drug in the assay.

Among the designed analogs, 4k, 4l, 4m, 4o, and 4p showed satisfactory and comparable docking results such as free binding energy and predicted inhibitory constant (Ki) as that of standard drug efavirenz and TNK-651. Docking results encourage us towards their synthesis and in vitro $\mathrm{RT}$ inhibition evaluation. In vitro evaluation of these compounds (4a, $\mathbf{4 b}, \mathbf{4 f}, \mathbf{4 g}$, $\mathbf{4 k}$, and $\mathbf{4 l}$ ) showed weak HIV-1 RT inhibitory activity at $20 \mu \mathrm{M}$ concentration. In this series of compounds $\mathbf{4 a}$ (2-(1,3-dioxo-3a,4-dihydro- $1 H$-isoindol-2( $3 H, 7 H, 7 \mathrm{aH})$-yl)- $N$-phenylacetamide) having un-substituted phenyl ring (mentioned as wing 2 in pharmacophore) showed 25\% inhibition of HIV-1 RT at tested concentration of $20 \mu \mathrm{M}$. Compound $4 \mathbf{f}$ (2-(1,3-dioxo-3a,4-dihydro-1H-isoindol-2(3H,7H,7aH)-yl)$\mathrm{N}$-m-tolylacetamide), having m-tolyl (3-methylphenyl) group as wing 2, inhibited $20 \%$ of HIV-1 RT at $20 \mu \mathrm{M}$ concentration. However, none of these compounds showed HIV-1 RT inhibition at $2 \mu \mathrm{M}$ concentration.

\section{Experimental}

All solvents and reagents purchased from Sigma (Bangalore, India) or Merck (NJ, USA) companies are used as received without further purification. Solvent system used throughout experimental work for running thin layer chromatography was ethyl acetate and hexane mixture (30:70) in order to monitor the reaction.

Melting points are uncorrected and were determined in open capillary tubes on a Precision Buchi B530 (Flawil, Switzerland) melting point apparatus containing silicon oil. IR spectra were recorded using a Jasco FTIR spectrophotometer (JASCO, Inc., USA). ${ }^{1} \mathrm{H}$ NMR spectra were recorded on a Bruker DPX-400 spectrometer (Bruker India Scientific Pvt. Ltd., Mumbai) using TMS as an internal standard (chemical shifts 
Table 2 Predicted molecular parameters of the synthesized compounds

\begin{tabular}{llllllll}
\hline Compound code & CLogP & Molecular weight & Number of HBA & Number of HBD & Solubility & Drug likeness & Drug score \\
\hline $4 \mathrm{a}$ & 1.10 & 284 & 5 & 1 & -2.45 & 2.29 & 0.7 \\
$4 \mathrm{~b}$ & 1.00 & 314 & 6 & 1 & -2.47 & 2.67 & 0.34 \\
$4 \mathrm{c}$ & 1.42 & 298 & 5 & 1 & -2.79 & 2.54 & 0.42 \\
$4 \mathrm{~d}$ & 1.72 & 318 & 5 & 1 & -3.19 & 4.71 & 0.69 \\
$4 \mathrm{e}$ & 1.00 & 314 & 6 & 1 & -2.47 & 3.65 & 0.43 \\
$4 \mathrm{f}$ & 1.42 & 298 & 5 & 1 & -2.79 & 3.56 & 0.71 \\
$4 \mathrm{~g}$ & 1.72 & 318 & 5 & 1 & -3.19 & 3.93 & 0.69 \\
$4 \mathrm{~h}$ & 1.00 & 314 & 6 & 1 & -2.47 & 4.05 & 0.72 \\
$4 \mathrm{i}$ & 1.42 & 298 & 5 & 1 & -2.79 & 4.07 & 0.71 \\
$4 \mathrm{j}$ & 1.72 & 318 & 5 & 1 & -3.19 & 4.17 & 0.55 \\
$4 \mathrm{k}$ & 0.97 & 329 & 8 & 1 & -2.91 & -11.4 & 0.28 \\
$4 \mathrm{l}$ & 0.97 & 329 & 8 & 1 & -2.91 & -1.34 & 0.27 \\
$4 \mathrm{~m}$ & 0.97 & 329 & 8 & 1 & -2.91 & -3.08 & 0.22 \\
$4 \mathrm{n}$ & 1.74 & 312 & 5 & 1 & -3.14 & 0.76 & -0.22 \\
40 & 1.74 & 312 & 5 & 5 & -3.14 & -3.53 & 3.93 \\
$4 \mathrm{p}$ & 2.03 & 332 & & 1 & 0.47 & 0.32 \\
\hline
\end{tabular}

in $\delta$ ). The ESMS were recorded on MICROMASS Quattro-II LCMS system (Waters Corporation, Milford, USA). Elemental analysis was performed on Vario EL III $\mathrm{M} / \mathrm{s}$ Elementar $\mathrm{C}, \mathrm{H}, \mathrm{N}$, and $\mathrm{S}$ analyzer (Elementar Analysensysteme $\mathrm{GmbH}$, Germany).

\section{General procedure for synthesis of the compounds} 3-(1,3-dioxo-3a,4-dihydro-1H-isoindol-2(3H,7H,7aH)-yl)-N(substituted phenyl) acetamides 4

To a solution of $3 \mathrm{a}, 4,7,7 \mathrm{a}$-tetrahydro- $\mathrm{H}$-isoindole-1,3 $(2 \mathrm{H})$-dione $(3)(2 \mathrm{mmol})$ in acetonitrile, potassium carbonate $(6 \mathrm{mmol})$ and corresponding 2-chloro- $N$-(substituted phenyl) acetamides $\mathbf{2}(\mathbf{a}-\mathbf{p})(2 \mathrm{mmol})$ were added and refluxed for $8 \mathrm{~h}$. On completion of the reaction as monitored by TLC, the contents were poured on crushed ice. Resulted precipitate was filtered, dried, and recrystallized from ethanol to obtain pure product 4.

\section{2-(1,3-dioxo-3a,4-dihydro-1H-isoindol-2(3H,7H,7aH)-yl)-N-} phenylacetamide (4a)

White solid (yield $84 \%, \mathrm{MP}=96^{\circ} \mathrm{C}$ to $\left.98^{\circ} \mathrm{C}\right)$. IR $\left(\mathrm{KBr}, \mathrm{cm}^{-1}\right.$ ): $3,271(\mathrm{~N}-\mathrm{H}), 1,776$, and 1,712 (C= O, isoindole), 1,693 ( $\mathrm{C}=$ $\mathrm{O}$, amide). $\mathrm{MS}\left(\mathrm{ES}^{+}\right): \mathrm{m} / z=285.6[M+1]$. Analytically calculated for $\mathrm{C}_{16} \mathrm{H}_{16} \mathrm{~N}_{2} \mathrm{O}_{3}$ (\%) C, 67.80; H, 5.25; N, 9.60. Found: C, 67.75; H, 5.30; N, 9.55.

\section{2-(1,3-dioxo-3a,4-dihydro-1H-isoindol-2(3H,7H,7aH)-yl)-N-(4-} methoxyphenyl)acetamide (4b)

White solid (yield $92 \%, \mathrm{MP}=102^{\circ} \mathrm{C}$ to $\left.104^{\circ} \mathrm{C}\right)$. IR $(\mathrm{KBr}$, $\left.\mathrm{cm}^{-1}\right)$ : 3,305 (N-H), 1,778, and 1,710 ( $\mathrm{C}=\mathrm{O}$, isoindole),<smiles>[R]c1ccc(NC(=O)CN2C(=O)C3CC=CCC3C2=O)cc1</smiles>

Scheme 1 Designed analogs synthesized using a synthetic protocol. (a) Triethylamine, dichloromethane, room temperature, 30 min; (b) $\mathrm{K}_{2} \mathrm{CO}_{3}$, acetonitrile, reflux, 7 to $8 \mathrm{~h}$. 


\begin{tabular}{llll}
$\begin{array}{l}\text { Table } 3 \text { HIV-1 RT inhibitory activity of synthesized } \\
\text { compounds }\end{array}$ & \multicolumn{2}{c}{ \%RT inhibition } \\
\hline $\begin{array}{llll}\text { Serial } \\
\text { number }\end{array}$ & $\begin{array}{l}\text { Compound } \\
\text { code }\end{array}$ & $\mathbf{2} \boldsymbol{\mu M}$ & $\mathbf{2 0} \boldsymbol{\mu M}$ \\
\hline 1 & $4 \mathrm{a}$ & $\mathrm{NA}$ & 25 \\
2 & $4 \mathrm{~b}$ & $\mathrm{NA}$ & 10 \\
3 & $4 \mathrm{c}$ & $\mathrm{NA}$ \\
4 & $4 \mathrm{~d}$ & $\mathrm{NA}$ & $\mathrm{NA}$ \\
5 & $4 \mathrm{e}$ & $\mathrm{NA}$ & $\mathrm{NA}$ \\
6 & $4 \mathrm{f}$ & $\mathrm{NA}$ & 20 \\
7 & $4 \mathrm{~g}$ & $\mathrm{NA}$ & 15 \\
8 & $4 \mathrm{~h}$ & $\mathrm{NA}$ & $\mathrm{NA}$ \\
9 & $4 \mathrm{i}$ & $\mathrm{NA}$ & $\mathrm{NA}$ \\
10 & $4 \mathrm{j}$ & $\mathrm{NA}$ & $\mathrm{NA}$ \\
11 & $4 \mathrm{k}$ & $\mathrm{NA}$ & 15 \\
12 & $4 \mathrm{l}$ & $\mathrm{NA}$ & 10 \\
13 & $4 \mathrm{~m}$ & $\mathrm{NA}$ & $\mathrm{NA}$ \\
14 & $4 \mathrm{n}$ & $\mathrm{NA}$ & $\mathrm{NA}$ \\
15 & $4 \mathrm{O}$ & $\mathrm{NA}$ & $\mathrm{NA}$ \\
16 & $4 \mathrm{p}$ & $\mathrm{NA}$ & $\mathrm{NA}$ \\
\hline $\mathrm{NA}$ & & &
\end{tabular}

NA indicates not active.

1,697 ( $\mathrm{C}=\mathrm{O}$, amide), 1,249 (C-O-C). Analytically calculated for $\mathrm{C}_{17} \mathrm{H}_{18} \mathrm{~N}_{2} \mathrm{O}_{4}$ (\%) $\mathrm{C}, 64.70 ; \mathrm{H}, 5.55 ; \mathrm{N}, 8.70$. Found: C, 64.75; H, 5.50; N, 8.65.

2-(1,3-dioxo-3a,4-dihydro-1H-isoindol-2(3H,7H,7aH)-yl)-N-ptolylacetamide (4c)

White solid (yield $82 \%, \mathrm{MP}=100^{\circ} \mathrm{C}$ to $\left.102^{\circ} \mathrm{C}\right)$. IR $(\mathrm{KBr}$, $\left.\mathrm{cm}^{-1}\right)$ : 3,408 (N-H), 1,772, and 1,712 (C=O, isoindole), 1,698 ( $\mathrm{C}=\mathrm{O}$, amide). ${ }^{1} \mathrm{H} \mathrm{NMR}\left(400 \mathrm{MHz}, \mathrm{CDCl}_{3}\right) 7.36$ (brs, 1H, NH), 7.32 (d, J=7.3 Hz, 2H, ArH), 7.10 (d, J= $7.1 \mathrm{~Hz}, 2 \mathrm{H}, \mathrm{ArH}), 5.96$ to $5.97(\mathrm{~m}, 2 \mathrm{H}, \mathrm{CH}=\mathrm{CH}), 4.27$ (s, $2 \mathrm{H}, \mathrm{CH}_{2}$ ), 3.21 to 3.23 (m, $2 \mathrm{H}, \mathrm{CH}-\mathrm{CH}$ ), 2.63 to 2.69 $(\mathrm{m}, 2 \mathrm{H}, \mathrm{CH}, \mathrm{CHH}), 2.30\left(\mathrm{~s}, 3 \mathrm{H}, \mathrm{CH}_{3}\right), 2.26$ to $2.28(\mathrm{~m}$, $2 \mathrm{H}, \mathrm{CH} H, \mathrm{CH} H)$. MS $\left(\mathrm{ES}^{+}\right): m / z=299.6[M+1]$. Analytically calculated for $\mathrm{C}_{17} \mathrm{H}_{18} \mathrm{~N}_{2} \mathrm{O}_{3}$ (\%) $\mathrm{C}, 68.60 ; \mathrm{H}$, 6.25; N, 9.70. Found: C, 68.65; H, 6.20; N, 9.65.

\section{$\mathrm{N}$-(4-chlorophenyl)-2-(1,3-dioxo-3a,4-dihydro-1H-isoindol-2 $(3 \mathrm{H}, 7 \mathrm{H}, 7 \mathrm{aH})$-yl)acetamide (4d)}

White solid (yield $84 \%, \mathrm{MP}=110^{\circ} \mathrm{C}$ to $112^{\circ} \mathrm{C}$ ). IR $(\mathrm{KBr}$, $\left.\mathrm{cm}^{-1}\right): 3,363(\mathrm{~N}-\mathrm{H}), 1,768$, and 1,706 ( $\mathrm{C}=\mathrm{O}$, isoindole), 1,698 ( $\mathrm{C}=\mathrm{O}$, amide), 689 (C-Cl). Analytically calculated for $\mathrm{C}_{16} \mathrm{H}_{15} \mathrm{ClN}_{2} \mathrm{O}_{3}$ (\%) C, 60.35; $\mathrm{H}, 4.60 ; \mathrm{N}, 8.85$. Found: $\mathrm{C}$, 60.40; H, 4.55; N, 8.90 .

2-(1,3-dioxo-3a,4-dihydro-1H-isoindol-2(3H,7H,7aH)-yl)-N-(3methoxyphenyl)acetamide (4e)

White solid (yield $78 \%, \mathrm{MP}=82^{\circ} \mathrm{C}$ to $84^{\circ} \mathrm{C}$ ). IR $\left(\mathrm{KBr}, \mathrm{cm}^{-1}\right)$ : $3,259(\mathrm{~N}-\mathrm{H}), 1,774$, and 1,712 ( $\mathrm{C}=\mathrm{O}$, isoindole), 1,703 ( $\mathrm{C}=$
O, amide), 1,234 (C-O-C). Analytically calculated for $\mathrm{C}_{17} \mathrm{H}_{18} \mathrm{~N}_{2} \mathrm{O}_{4}$ (\%) C, 64.80; H, 5.50; N, 8.65. Found: C, 64.75; $\mathrm{H}, 5.55 ; \mathrm{N}, 8.70$.

\section{2-(1,3-dioxo-3a,4-dihydro-1H-isoindol-2(3H,7H,7aH)-yl)-N-m-} tolylacetamide (4f)

White solid (yield $76 \%, \mathrm{MP}=86^{\circ} \mathrm{C}$ to $88^{\circ} \mathrm{C}$ ). IR $\left(\mathrm{KBr}, \mathrm{cm}^{-1}\right.$ ): 3,342 (N-H), 1,776, and 1,712 ( $\mathrm{C}=\mathrm{O}$, isoindole), 1,682 $(\mathrm{C}=$ $\mathrm{O}$, amide). Analytically calculated for $\mathrm{C}_{17} \mathrm{H}_{18} \mathrm{~N}_{2} \mathrm{O}_{3}$ (\%) $\mathrm{C}$, 68.20; H, 6.35; N, 9.30. Found: C, 68.25; H, 6.30; N, 9.25.

\section{$\mathrm{N}$-(3-chlorophenyl)-2-(1,3-dioxo-3a,4-dihydro-1H-isoindol-2} $(3 \mathrm{H}, 7 \mathrm{H}, 7 \mathrm{aH})$-yl)acetamide $(4 \mathrm{~g})$

White solid (yield $78 \%, \mathrm{MP}=92^{\circ} \mathrm{C}$ to $\left.94^{\circ} \mathrm{C}\right)$. IR $\left(\mathrm{KBr}, \mathrm{cm}^{-1}\right.$ ): 3,290 (N-H), 1,768, and 1,712 ( C = O, isoindole), 1,697 ( $\mathrm{C}=$ $\mathrm{O}$, amide), $678(\mathrm{C}-\mathrm{Cl})$. Analytically calculated for $\mathrm{C}_{16}$ $\mathrm{H}_{15} \mathrm{ClN}_{2} \mathrm{O}_{3}$ (\%) C, 60.50; H, 4.20; N, 8.90. Found: C, 60.45; $\mathrm{H}, 4.15$; N, 8.95.

2-(1,3-dioxo-3a,4-dihydro-1H-isoindol-2(3H,7H,7aH)-yl)-N-(2methoxyphenyl)acetamide (4h)

White solid (yield $76 \%, \mathrm{MP}=78^{\circ} \mathrm{C}$ to $\left.80^{\circ} \mathrm{C}\right)$. IR $\left(\mathrm{KBr}, \mathrm{cm}^{-1}\right)$ : 3,345 (N-H), 1,774, and 1,712 ( $\mathrm{C}=\mathrm{O}$, isoindole), 1,703 ( $\mathrm{C}=$ $\mathrm{O}$, amide), 1,242(C-O-C). Analytically calculated for $\mathrm{C}_{17}$ $\mathrm{H}_{18} \mathrm{~N}_{2} \mathrm{O}_{4}$ (\%) C, 64.75; H, 5.80; N, 8.55. Found: C, 64.80; H, $5.75 ; \mathrm{N}, 8.60$.

\section{2-(1,3-dioxo-3a,4-dihydro-1H-isoindol-2(3H,7H,7aH)-yl)-N-o-} tolylacetamide (4i)

White solid (yield $74 \%, \mathrm{MP}=92^{\circ} \mathrm{C}$ to $94^{\circ} \mathrm{C}$ ). IR $\left(\mathrm{KBr}, \mathrm{cm}^{-1}\right.$ ). $3,265(\mathrm{~N}-\mathrm{H}), 1,772$, and 1,705 ( $\mathrm{C}=\mathrm{O}$, isoindole), 1,694 ( $\mathrm{C}=$ $\mathrm{O}$, amide). Analytically calculated for $\mathrm{C}_{17} \mathrm{H}_{18} \mathrm{~N}_{2} \mathrm{O}_{3}$ (\%) $\mathrm{C}$, 68.55; H, 6.25; N, 9.40. Found: C, 68.50; H, 6.30; N, 9.45.

\section{$\mathrm{N}$-(2-chlorophenyl)-2-(1,3-dioxo-3a,4-dihydro-1H-isoindol-2 (3H,7H,7aH)-yl)acetamide (4j)}

White solid (yield $76 \%, \mathrm{MP}=88^{\circ} \mathrm{C}$ to $90^{\circ} \mathrm{C}$ ). IR $\left(\mathrm{KBr}, \mathrm{cm}^{-1}\right.$ ): $3,302(\mathrm{~N}-\mathrm{H}), 1,784$, and 1,702 $(\mathrm{C}=\mathrm{O}$, isoindole $), 1,676(\mathrm{C}=$ $\mathrm{O}$, amide), 697 (C-Cl). Analytically calculated for $\mathrm{C}_{16}$ $\mathrm{H}_{15} \mathrm{ClN}_{2} \mathrm{O}_{3}$ (\%) C, 60.10; H, 4.50; N, 8.95. Found: C, 60.15; $\mathrm{H}, 4.45$; N, 8.90.

2-(1,3-dioxo-3a,4-dihydro-1H-isoindol-2(3H,7H,7aH)-yl)-N-(4nitrophenyl)acetamide (4k)

Yellow solid (yield $86 \%, \mathrm{MP}=125^{\circ} \mathrm{C}$ to $127^{\circ} \mathrm{C}$ ). IR $(\mathrm{KBr}$, $\left.\mathrm{cm}^{-1}\right)$ : 3,325 (N-H), 1,779, and 1,710 (C=O, isoindole), 1,686 ( $\mathrm{C}=\mathrm{O}$, amide), 1,542, 1,322 $\left(\mathrm{C}-\mathrm{NO}_{2}\right)$. Analytically calculated for $\mathrm{C}_{16} \mathrm{H}_{15} \mathrm{~N}_{3} \mathrm{O}_{5}$ (\%) $\mathrm{C}, 58.60 ; \mathrm{H}, 4.70 ; \mathrm{N}$, 12.55. Found: C, 58.55; H, 4.65; N, 12.60 .

2-(1,3-dioxo-3a,4-dihydro-1H-isoindol-2(3H,7H,7aH)-yl)-N-(3nitrophenyl)acetamide (4l)

Yellow solid (yield $70 \%, \mathrm{MP}=112^{\circ} \mathrm{C}$ to $\left.114^{\circ} \mathrm{C}\right)$. IR $(\mathrm{KBr}$, $\left.\mathrm{cm}^{-1}\right)$ : 3,338 (N-H), 1,774, and 1,712 (C= O, isoindole), 
1,693 ( $\mathrm{C}=\mathrm{O}$, amide), 1,537, 1,327 $\left(\mathrm{C}-\mathrm{NO}_{2}\right)$. Analytically calculated for $\mathrm{C}_{16} \mathrm{H}_{15} \mathrm{~N}_{3} \mathrm{O}_{5}$ (\%) C, 58.45; H, 5.05; N, 12.45. Found: C, 58.50; H, 5.10; N, 12.50 .

\section{2-(1,3-dioxo-3a,4-dihydro-1H-isoindol-2(3H,7H,7aH)-yl)-N-(2- nitrophenyl)acetamide $(4 \mathrm{~m})$}

Yellow solid (yield $64 \%, \mathrm{MP}=102^{\circ} \mathrm{C}$ to $\left.104^{\circ} \mathrm{C}\right)$. IR $(\mathrm{KBr}$, $\left.\mathrm{cm}^{-1}\right): 3,331(\mathrm{~N}-\mathrm{H}), 1,774$, and 1,714 $(\mathrm{C}=\mathrm{O}$, isoindole), 1,698 ( $\mathrm{C}=\mathrm{O}$, amide), 1,531, 1,336 $\left(\mathrm{C}-\mathrm{NO}_{2}\right)$. Analytically calculated for $\mathrm{C}_{16} \mathrm{H}_{15} \mathrm{~N}_{3} \mathrm{O}_{5}(\%) \mathrm{C}, 58.65 ; \mathrm{H}, 4.80 ; \mathrm{N}$, 12.50. Found: C, 58.70; H, 4.85; N, 12.55 .

\section{$\mathrm{N}$-(2,4-dimethylphenyl)-2-(1,3-dioxo-3a,4-dihydro-1H- isoindol-2(3H, $7 \mathrm{H}, 7 \mathrm{aH})$-yl)acetamide $(4 \mathrm{n})$}

White solid (yield $86 \%, \mathrm{MP}=106^{\circ} \mathrm{C}$ to $\left.108^{\circ} \mathrm{C}\right)$. IR $(\mathrm{KBr}$, $\left.\mathrm{cm}^{-1}\right): 3,284(\mathrm{~N}-\mathrm{H}), 1,782$, and 1,712 $(\mathrm{C}=\mathrm{O}$, isoindole $)$, $1,672\left(\mathrm{C}=\mathrm{O}\right.$, amide). Analytically calculated for $\mathrm{C}_{18}$ $\mathrm{H}_{20} \mathrm{~N}_{2} \mathrm{O}_{3}$ (\%) C, 69.45; H, 6.60; N, 8.75. Found: C, 69.40; $\mathrm{H}, 6.65 ; \mathrm{N}, 8.70$.

\section{$\mathrm{N}$-(3,4-dimethylphenyl)-2-(1,3-dioxo-3a,4-dihydro-1H- isoindol-2(3H,7H,7aH)-yl)acetamide (4o)}

White solid (yield $80 \%, \mathrm{MP}=110^{\circ} \mathrm{C}$ to $\left.112^{\circ} \mathrm{C}\right)$. IR $(\mathrm{KBr}$, $\left.\mathrm{cm}^{-1}\right): 3,286(\mathrm{~N}-\mathrm{H}), 1,778$, and 1,708 $(\mathrm{C}=\mathrm{O}$, isoindole), $1,697(\mathrm{C}=\mathrm{O}$, amide). Analytically calculated for $\mathrm{C}_{18} \mathrm{H}_{20} \mathrm{~N}_{2} \mathrm{O}_{3}$ (\%) C, 69.65; H, 6.80; N, 8.60. Found: C, 69.60; H, 6.85; N, 8.65.

\section{$\mathrm{N}$-(2-chloro-3-methylphenyl)-2-(1,3-dioxo-3a,4-dihydro-1H- isoindol-2(3H,7H,7aH)-yl)acetamide $(4 p)$}

White solid (yield $84 \%, \mathrm{MP}=118^{\circ} \mathrm{C}$ to $\left.120^{\circ} \mathrm{C}\right)$. IR $(\mathrm{KBr}$, $\left.\mathrm{cm}^{-1}\right): 3,356(\mathrm{~N}-\mathrm{H}), 1,786$, and 1,714 $(\mathrm{C}=\mathrm{O}$, isoindole), 1,695 ( $\mathrm{C}=\mathrm{O}$, amide), $694(\mathrm{C}-\mathrm{Cl})$. Analytically calculated for $\mathrm{C}_{17} \mathrm{H}_{17} \mathrm{ClN}_{2} \mathrm{O}_{3}$ (\%) C, 61.50; H, 5.35; N, 8.65. Found: C, 61.55; H, 5.40; N, 8.70.

\section{HIV-1 RNA-dependent DNA polymerase activity assay} Poly(rA)/oligo(dT) was used as a template for the RNAdependent DNA polymerase reaction by HIV-1 RT, either wild type or carrying the mutations. For the activity assay, $25 \mu$ f final reaction volume contained TDB buffer (50 mM Tris- $\mathrm{HCl}$ (pH 8.0), $1 \mathrm{mM}$ dithiothreitol, $0.2 \mathrm{mg} /$ $\mathrm{ml}$ bovine serum albumin, $2 \%$ glycerol), $10 \mathrm{mM} \mathrm{MgCl}_{2}$, $0.5 \mathrm{mg}$ of poly(rA)/oligo(dT)10:1 (0.3 mM 3'-OH ends), and $10 \mathrm{mM}{ }^{3}[\mathrm{H}]-$ dTTP $(1 \mathrm{Ci} / \mathrm{mmol})$, and was introduced into tubes containing aliquots of different enzyme concentrations (5 to $10 \mathrm{nM} \mathrm{RT}$ ). After incubation at $37^{\circ} \mathrm{C}$ for indicated time, $20 \mu \mathrm{L}$ from each reaction tube were spiked on glass fiber filters GF/C and immediately immersed in 5\% ice-cold trichloroacetic acid (TCA) (AppliChem $\mathrm{GmbH}$, Darmstadt). Filters were washed three times with 5\% TCA and once with ethanol for 5 min, then dried, and finally added with EcoLume scintillation cocktail (ICN, Research Products Division, Costa
Mesa, CA, USA) to detect the acid precipitable radioactivity by PerkinElmer Trilux MicroBeta 1450 Counter (Waltham, MA, USA).

\section{Conclusion}

All the synthesized 3-(1,3-dioxo-3a,4-dihydro- $1 \mathrm{H}$-isoindol-2(3H,7H,7aH)-yl)- $N$-(substituted phenyl) acetamide 4(a-p) analogs were evaluated for HIV-1 reverse transcriptase inhibitor activity.

Among these synthesized compounds, 4a, 4b, 4f, 4g, 4k, and $4 \mathbf{l}$ showed weak HIV-1 RT inhibitor activity at $20 \mu \mathrm{M}$ concentration. There was no correlation observed between molecular modeling and in vitro studies for these synthesized compounds.

\section{Abbreviations}

AIDS: Acquired immune deficiency syndrome; HIV: Human immunodeficiency virus; RT: Reverse transcriptase; HAART: Highly active anti-retroviral therapy; NRTI: Nucleoside reverse transcriptase inhibitor; NNRTI: Non-nucleoside reverse transcriptase inhibitor; PI: Protease inhibitor; NNIBP: Non-nucleoside inhibitory binding pocket.

\section{Competing interests}

The authors declare that they have no competing interests.

\section{Acknowledgements}

We thank SAIF, Punjab University for providing NMR spectra and SAIF-CDRI for mass spectra. We greatly acknowledge Nicolas Cremor, Pittsburgh University, USA for HIV-1 RT screening of our compounds. One of the authors, AP, acknowledges the financial support from UGC-BSR, New Delhi in the form of Junior Research Fellowship.

\section{Author details}

'Department of Pharmacy, Birla Institute of Technology \& Science, Pilani Rajasthan 333031, India. ${ }^{2}$ Department of Pharmaceutical Sciences, Birla Institute of Technology, Mesra-Jharkhand 835215, India.

\section{Received: 14 May 2013 Accepted: 22 July 2013}

Published: 22 August 2013

\section{References}

1. UNAIDS (2012) Report on the global AIDS epidemic 2012. http://www. unaids.org/. Accessed 21 Feb 2013

2. Sun LQ, Zhu L, Qian K, Qin B, Huang L, Chen CH, Lee KH, Xie L (2012) Design, Synthesis and preclinical evaluations of novel 4-substituted 1,5-diarylanilines as potent HIV-1 non-nucleoside reverse transcriptase inhibitor (NNRTI) drug candidates. J Med Chem 55:7219-7229

3. Chong P, Sebahar P, Youngman M, Garrido D, Zhang H, Eugene L, Robert ST, Wang NL, Ferris RG, Edelstein M, Weaver K, Mathis A, Peat A (2012) Rational design of potent non-nucleoside inhibitors of HIV-1 reverse transcriptase. J Med Chem 55:10601-10609

4. Bethune MP (2010) Non-nucleoside reverse transcriptase inhibitors (NNRTIs), their discovery, development and use in the treatment of HIV-1 infection: a review of the last 20 years (1989-2009). Antiviral Res 85:75-90

5. Li D, Zhan P, Clercq ED, Liu X (2012) Strategies for the design of HIV-1 non-nucleoside reverse transcriptase inhibitors: lessons from the development of seven representative paradigms. J Med Chem 55:3595-3613

6. Paul AJ, Janssen PAJ, Lewi PJ, Arnold E, Daeyaert F, Jonge $M$, Heeres J, Koymans L, Vinkers M, Guillemont J, Pasquier E, Kukla M, Ludovici D, Andries K, Béthune MPD, Pauwels R, Das K, Clark AD, Frenkel YV, Hughes SH, Medaer B, Knaep FD, Bohets H, Clerck FD, Lampo A, Williams P, Stoffels P (2005) In search of a novel anti-HIV drug: multidisciplinary coordination in the discovery of 4-[[4-[[4-[(1E)-2-cyanoethenyl]-2,6-dimethylpheny|]amino]-2-pyrimidinyl]amino] benzonitrile (R278474, rilpivirine). J Med Chem 48:1901-1909

7. Hopkins AL, Ren J, Esnouf RM, Willcox BE, Jones EY, Ross C, Miyasaka T, Walker RT, Tanaka H, Stammers DK, Stuart DI (1996) Complexes of HIV-1 reverse transcriptase with inhibitors of the HEPT series reveal 
conformational changes relevant to the design of potent non-nucleoside inhibitors. J Med Chem 39:1589-1600

8. Lima LM, Castro P, Machado AL, Fraga CAM, Lugnier C, Moraesc VLGD, Barreiro EJ (2002) Synthesis and anti-inflammatory activity of phthalimide derivatives, designed as new thalidomide analogues. Bioorg Med Chem 10:3067-3073

9. Dos Santos JL, Lanaro C, Chelucci RC, Gambero S, Bosquesi PL, Reis JS, Lima LM, Cerecetto H, Gonzalez M, Costa FF, Chung MC (2012) Design, synthesis and pharmacological evaluation of novel hybrid compounds to treat sickle cell disease symptoms. Part Il: furoxan derivatives. J Med Chem 55:7583-7592

10. Bhambi D, Salvi VK, Bapna A, Pemawat G, Talesara GL (2009) Synthesis and antimicrobial evaluation of some alkoxyphthalimide derivatives of naphthyridine. Indian J Chem 48B:697-704

11. Ranise A, Spallarossa A, Schenone S, Bruno O, Bondavalli F, Vargiu L, Marceddu T, Mura M, Colla PL, Pani A (2003) Design, synthesis, structure-activity relationship and molecular modeling studies of acylthiocarbamates: a novel series of potent non-nucleoside HIV-1 reverse transcriptase inhibitors structurally related to phenethylthiazolylthiourea derivatives. J Med Chem 46:768-781

12. Ranise A, Spallarossa A, Cesarini S, Bondavalli F, Schenone S, Bruno O, Menozzi G, Fossa P, Mosti L, Colla ML, Sanna G, Murreddu M, Collu G, Busonera B, Marongiu ME, Pani A, Colla PL, Loddo R (2005) Structure-based design, parallel synthesis, structure-activity relationship and molecular modeling studies of thiocarbamates, new potent non-nucleoside HIV-reverse transcriptase inhibitor isosteres of phenethylthiazolylthiourea derivatives. J Med Chem 48:3858-3873

13. Verschueren WG, Dierynck I, Amssoms KIE, Hu L, Boonants PMJG, Pille GME, Daeyaert FFD, Hertogs K, Surleraux DLNG, Wigerinck PBTP (2005) Design and optimization of tricyclic phthalimide analogues as novel inhibitors of HIV-1 integrase. J Med Chem 48:1930-1940

14. Lindstrom W, Morris GM, Weber C, Huey R (2008) AutoDock 4.2. The Scripps Research Institute, Molecular Graphics Laboratory, California, USA. http:// autodock.scripps.edu. Accessed 21 Feb 2013

15. Schuettelkopf AW, Aalten DMFV (2004) PRODRG: a tool for high-throughput crystallography of protein-ligand complexes. Acta Crystallogrphica D60:1355-1363

16. Molinspiration Cheminformatics (1986) Molinspiration. http://www. molinspiration.com/cgi-bin/properties. Accessed 22 Feb 2013

17. Organic Chemistry Portal () Molecular property explorer, OSIRIS. http:// www.organic-chemistry.org/prog/peo. Accessed 23 Feb 2013

18. Ganguly S, Murugesan S, Maga G (2009) Synthesis, evaluation and molecular modeling studies of some novel tetrahydroisoquinoline derivatives targeted at the HIV-1 reverse transcriptase. Indian J Heterocycl Chem 18:357-360

19. Murugesan S, Ganguly S, Maga G (2010) Synthesis, evaluation and molecular modeling studies of some novel 3-(3,4-dihydroisoquinolin-2(1H)-yl)-N(substituted phenyl) propanamides as HIV-1 non-nucleoside reverse transcriptase inhibitors. J Chem Sci 122:169-176

20. Cremer NS, Hamamouch N, Felix AS, Velazquez S, Balzarini J, Camarasa MJ (2006) Structure activity relationship of [2,'5'-bis-O-(tert-butyl dimethyl silyl)$\beta$-D-ribofuranosyl]-3'-spiro-5-(4-amino-1,2-oxathiole-2,2-dioxide) thymine derivatives as inhibitors of HIV-1 reverse transcriptase dimerization. J Med Chem 49:4834-4841

doi:10.1186/2191-2858-3-8

Cite this article as: Penta et al:: Design and synthesis of tetrahydrophthalimide derivatives as inhibitors of HIV-1 reverse transcriptase. Organic and Medicinal Chemistry Letters 2013 3:8.

\section{Submit your manuscript to a SpringerOpen ${ }^{\circ}$ journal and benefit from:}

- Convenient online submission

- Rigorous peer review

- Immediate publication on acceptance

- Open access: articles freely available online

- High visibility within the field

- Retaining the copyright to your article

Submit your next manuscript at $\gg$ springeropen.com 